\title{
Comparison of Measurement Based and Site Specific Ray Based Microcellular Path Loss Predictions*
}

\author{
Leonard Piazzi, George Liang, Henry L. Bertoni and Seongcheol Kim* \\ Center for Advanced Technology in Telecommunications \\ Polytechnic University \\ Brooklyn, NY 11201
}

\begin{abstract}
The performance of site speciffc ray tracing methods based on UTD to predict the path loss are compared against measurement based models and measurements. An empirical formulation based on a simple least squares regression fit from one site is used as a prediction tool at another site having similar building environment. Comparing the accuracy of both types of prediction models, it is found that the site specific method provides an improvement over the traditional method of measurement based path loss prediction.
\end{abstract}

\section{INTRODUCTION}

$\mathrm{T}$ HE development of mature systems for Personal Communication Services (PCS) are envisioned to employ base station antennas at lamp post heights covering distances of $1 \mathrm{~km}$ or less. Prediction of the path loss for such low low antennas is required to determine the actual shape of individual cells, and to evaluate interference with neighboring cells. Typically predictions are made using empirical models derived from measurement data collected in various building environments. In these models the variation of the average signal strength is given as a simple inverse power law of the form $\frac{A}{R^{n}}$, where $R$ is the radial distance from the base station, $A$ is the signal level at 1 meter, and the value of the exponent $n$, can range from 2 to 6 [1]. The accuracy of such models are limited, with significant mean error and standard deviations ranging up to $8 \mathrm{~dB}$ [2]. While the model can be modified to account for general environmental factors such as terrain, or suburban or rural environments, it yields circular coverage areas, which is not realistic.

In this study, measurements taken in Trenton, NJ and Rosslyn, VA are used as the basis in the simple model outlined above to create a predictor algorithm for other areas of Trenton and Rosslyn, respectively. In these two cities we are also able to accounted for the height, shape and location of individual buildings to make theoretical site specific predictions of the sector averaged path loss using ray methods embodying the uniform theory of diffraction (UTD) [3]. In general the theoretical site specific predictions are more accurate then the predictions based upon a simple regression fit to measurements.

\footnotetext{
*This work was supported in part by a Motorola Partnership in Research Grant and in part by a grant from the New York State Science and Technology Foundation.

${ }^{*} \mathrm{~S}$. Kim is now with AT\&T, Holmdel, N.J.

$0-7803-3300-4 / 96 \$ 5.00 @ 1996$
}

\section{Measurement Areas}

The measurements reported in this work were made in Trenton, NJ and Rosslyn, VA. In Trenton, the areas studied were in residential neighborhoods consisting primarily of two story houses, typically 24 feet tall. The transmitting system for this area consisted of two antennas at 18 and 36 feet from the ground, with an operating frequency of 1800 $\mathrm{MHz}$. The two antennas heights were therefore above and below the surrounding buildings respectively. A street map showing the measurement points at Site B, as provided by an ETAC positioning system, is shown in Figure 1, along with building footprints. The original map from which Fig-

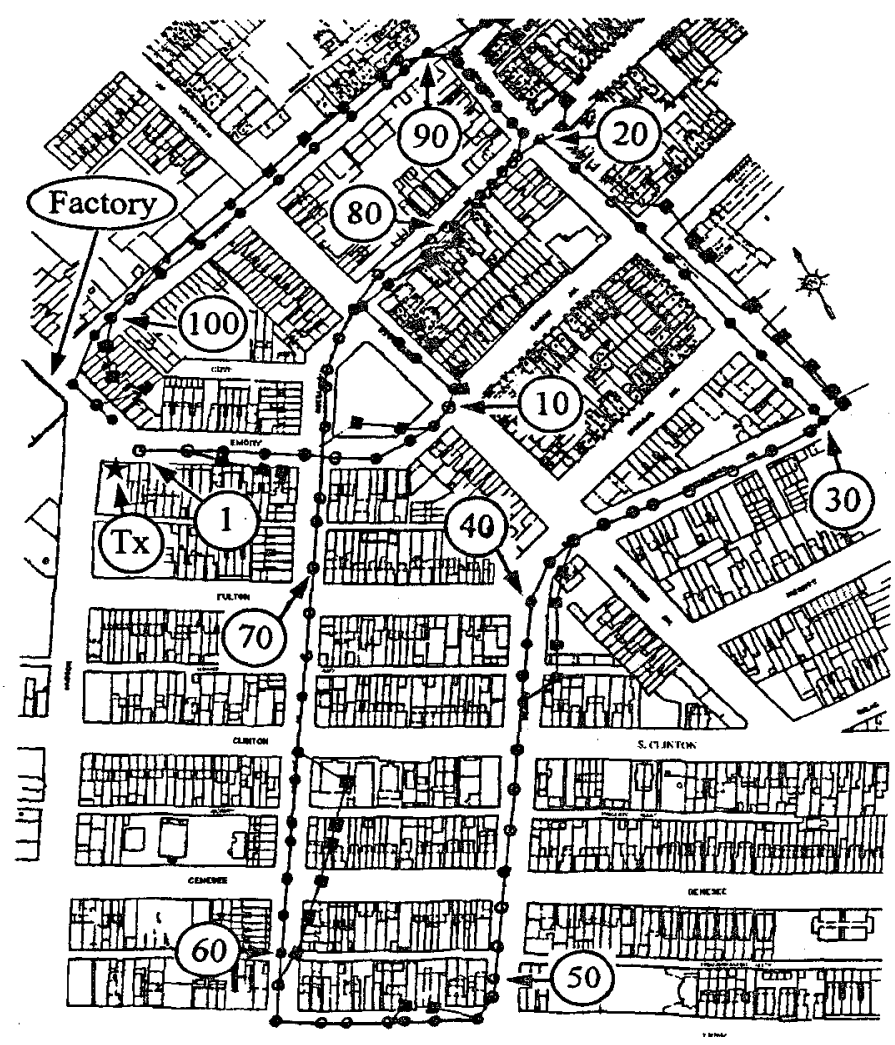

Fig. 1. Building Layout and Receiver Locations for Site B in Trenton, NJ.

ure 1 was taken also showed the building heights in stories.

The buildings in Rosslyn are primarily office/commercial structures, with typical heights of ten to thirty stories. Once again the position of the measurement point or mobile is recorded as the mobile is driven about the streets surrounding the base station. Figure 2 indicates the transmitter and receiver locations on the streets of Rosslyn, and 


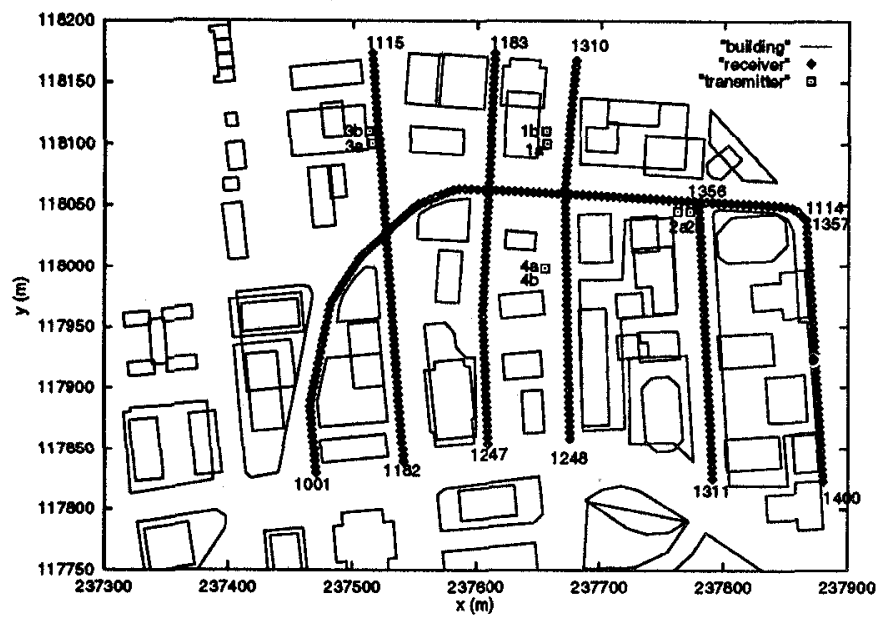

Fig. 2. Measurement Points and Building Footprints for Rosslyn, VA.

the building footprints. For this work we will only examine the measurements at a frequency of $1900 \mathrm{MHz}$, and a base station antenna height of $10 \mathrm{~m}$.

\section{MEASUREMENTS}

It is common to plot the measured signal strength as a function of the logarithm of the radial distance from the mobile to the base station, and to construct a straight line approximation using the least squares fit. For the 36 foot antenna in Trenton at Site B, the data and least squares fit are shown in Figure 3. In this case the slope of the least squares fit line gives a path loss index of $n=3.9$, and the

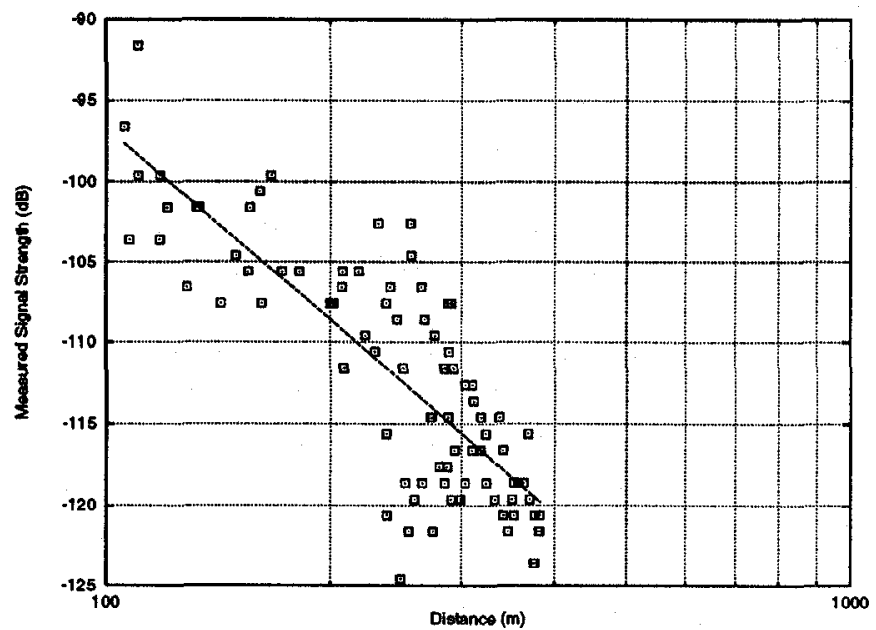

Fig. 3. Measurements with Applied Fit for the High Base Station Antenna at Site B

standard deviation between the fit and the measurements is $4.2 \mathrm{~dB}$.

The regression fit in Figure 3 for Site $B$ may be used as a predictor for the high antenna at a different area of Trenton having similar buildings, and referred to as Site A. Figure 4 shows the measurements taken at Site $A$, and the fit from Site B. The average error between the fitted line from Site B and the measurement is $-4.8 \mathrm{~dB}$ and the standard deviation

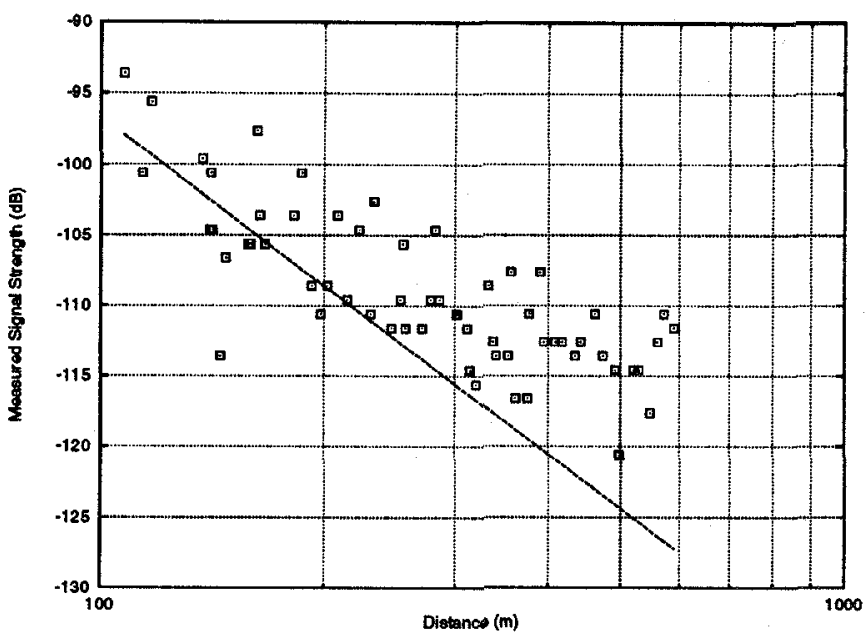

Fig. 4. Measurements with Line Fit of Site B for the High Base Station Antenna at Site A

is $5.0 \mathrm{~dB}$. These findings show that predictions based upon a simple regression fit to the measurement in one area can have a considerable average error and standard deviation when applied to measurements in another area with the same type of buildings. A similar discrepancy was found from measurements made using the base station antenna at 18 feet. We will show that the theoretical predictions of this work outperform the above predictions at Site A.

Figure 5 shows a scatter plot of the measured signal strength in Rosslyn versus the logarithm of the radial dis-

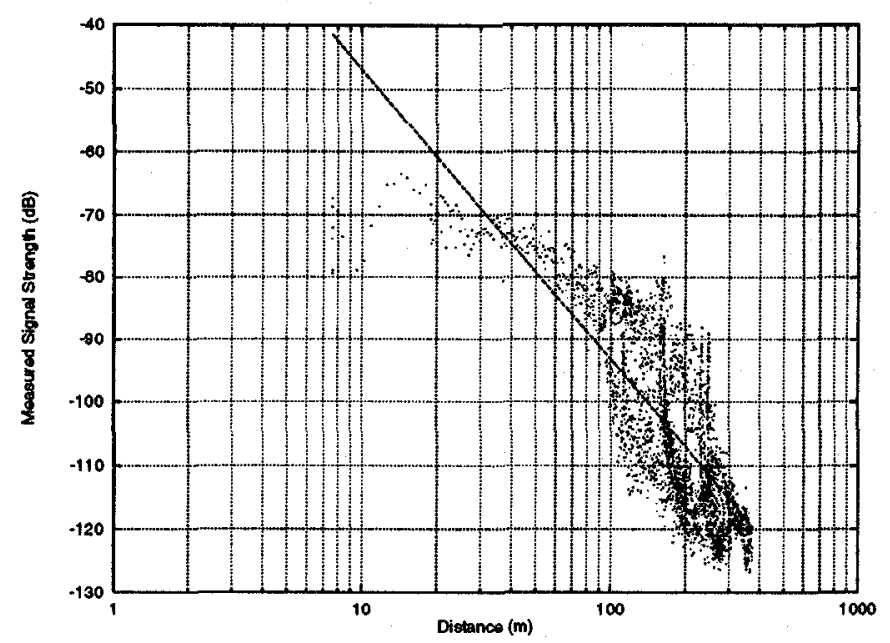

Fig. 5. Measurements with Applied Fit for the Base Station Antenna at Site $2 \mathrm{~b}$ in Rosslyn

tance between the mobile and the base station at location tx2b in Figure 2. The line represents a least square regression fit of the data and has a slope index of $n=4.6$. When the fit line is compared with its own data the standard deviation is found to be $8.8 \mathrm{~dB}$.

Figure 6 shows the plot of the fit line from the data of tx $2 b$ plotted against the measurements for the base station located at tx1a in Figure 2, which is located one block over from base station location tx $2 \mathrm{~b}$. When the regression fit 


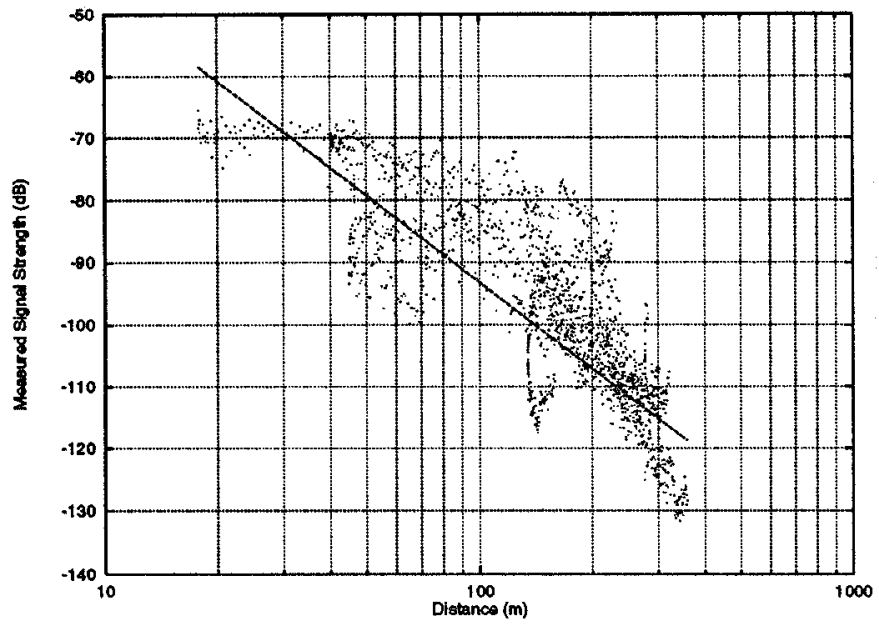

Fig. 6. Measurements with Line Fit of Site $2 b$ for the Base Station Antenna at Site 1a in Rosslyn

line from tx $2 \mathrm{~b}$ is used as a predictor at site tx1a the average error is $-3.4 \mathrm{~dB}$ and the standard deviation is $8.7 \mathrm{~dB}$. Since both transmitters are located in the same building environment and are separated by only one block, it would seems reasonable to expect a smaller average error, which represents the systematic error of the empirical model.

\section{Site Specific Predictions}

In the UHF band the signals traveling between the base station and mobile do so by propagating over and around intervening buildings by reflection and diffraction processes. Propagation prediction programs developed at Polytechnic University based on ray optics are is used to predict the sector average signal. A infinite number of rays can exist between the transmitter and receiver. Therefore in order to have a manageable computational problem it is necessary to trace only those rays that are the significant contributors at each receiver point.

Certain classes of rays will dominate depending on the relative height of the base station antenna to the surrounding building environment. When the transmitter is at or above the building rooftops the rays which multiply diffract over the buildings, in combination with reflections near the transmitter or receiver, are thought to be the dominate paths. When the base station antenna is significantly below the rooftops the signal arrives at the receiver primarily from ray paths which have undergone one or two diffractions at a vertical building corner, in combination with reflections from the building walls. In the region between these two cases where the antenna is near the rooftops of the surrounding buildings, or in the case where the base station is located in a heterogeneous environment of high and low rise buildings, all ray classes will contribute to the predicted signal level at a receiver.

In Trenton, signal contributions for both rays that propagate over and around buildings are considered. Figure 7 illustrates the predicted and measured values of received signal for the high antenna at Site A, which are plotted ver-

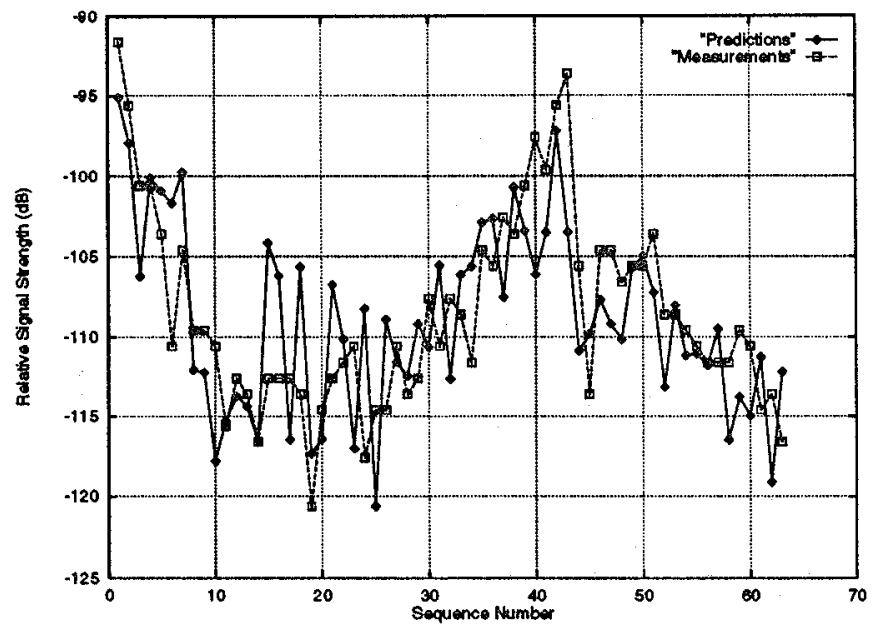

Fig. 7. Comparison of Theoretical Site Specific Predictions and Measurements for the High Antenna at Site A

sus position or sequence, number which indicate position along the drive path as shown in Figure 1. Good agreement is seen between predictions and measurements, with an average difference between them of $-0.4 \mathrm{~dB}$ and a standard deviation of $4.5 \mathrm{~dB}$. In this case only contributions from paths lying directly over the buildings, and paths involving reflections near to the mobile were considered. The theoretical predictions have a considerably smaller average error then the predictions shown in Figure 3 for Site A that were made using the slope intercept model based on Site $\mathrm{B}$ measurements. The standard deviation of the error is nearly the same as found for the least squares fit at Site B itself.

When the base station antenna is moved below the rooftops there are additional paths not considered in the high antenna case. In addition to the paths directly over the buildings, the paths including reflections near the mobile's position, and the paths involving diffraction at a vertical edge, we now include paths having a single reflection near the base station before propagation over the buildings to the mobile. Also, the signal contributions due to propagation around buildings must be included. No single ray type is consistently dominant at all mobile positions.

Figure 8 shows the predictions and measurements versus sequence number for the low antenna at Site A. The predictions are close to the measurements except at locations 1317 and 57-64, where the predictions result are significantly pessimistic. The average difference between the predictions and measurements is $-0.7 \mathrm{~dB}$, while the standard deviation is $4.9 \mathrm{~dB}$. These results are again consistent with the values obtained for the high antenna case. It should be noted that had propagation around buildings been neglected, the average difference would be $-5.1 \mathrm{~dB}$. Therefore, when the base station antenna is moved below the rooftops, even in residential areas, it is necessary to consider propagation around the buildings to obtain accurate results. The dielectric permittivity, $\epsilon_{r}$, used to calculate the reflection coefficients from the buildings is 3 in this case. Similar results 


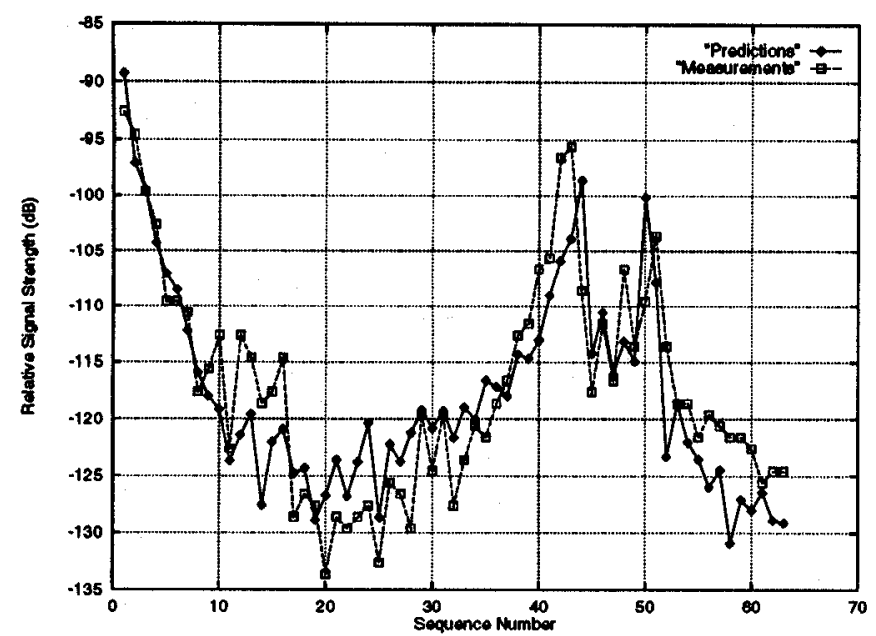

Fig. 8. Comparison of Theoretical Site Specific Predictions and Measurements for the Low Antenna at Site A

were obtained for predictions at site $B$, and are summarized in Table II

In Rosslyn the ten meter high base station antenna is surrounded by buildings that are typically tens of meters higher than the base station. In this high rise environment propagation over the buildings is considered to be a negligible contribution to the overall signal strength at the receiver. Therefore the computational problem can be reduce to a two dimensional problem where the rays are traced in the horizontal plane and the buildings are considered to be infinitely high. With this algorithm rays that are reflected from building walls, diffracted from building corners and rays involving combinations of these two processes are accounted for.

The theoretical site specific predictions versus measurements for Site 1a in Rosslyn is shown in Figure 9. For this case, when modeling the reflections from building walls the

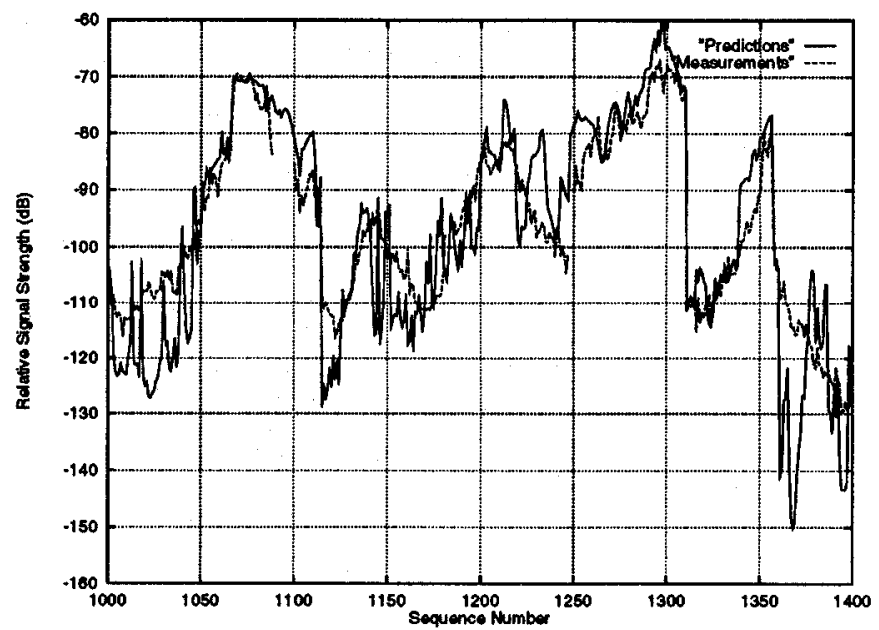

Fig. 9. Comparison of Theoretical Site Specific Predictions and Measurements for the Base Station Antenna at Site 1a in Rosslyn

dielectric permittivity is 12 . The predictions show good agreement with the measurements and follows the major trends. The predictions generally become more pessimistic as a receiver point moves deeper into the building shadows. The average difference between the predictions and the measurements is $-0.8 \mathrm{~dB}$ with a standard deviation of $9.4 \mathrm{~dB}$. Recall that the average difference from the empirical model of transmitter $2 \mathrm{~b}$ was $-3.4 \mathrm{~dB}$, and the standard deviation was $8.7 \mathrm{~dB}$. Therefore the theoretical site specific predictions yielded a smaller average error than the empirical model, with comparable standard deviations.

\section{A. Dielectric Constant of Building Walls}

Reflection from a building wall is typically approximated by the reflection coefficient of an infinite dielectric half space, whose reflection coefficient for a vertically polarized electric field is given by,

$$
\Gamma(\theta)=\frac{\cos \theta-a \sqrt{\epsilon_{r}-\sin ^{2} \theta}}{\cos \theta+a \sqrt{\epsilon_{r}-\sin ^{2} \theta}}
$$

The reflection coefficient is therefore a function of incident angle, $\theta$ and the dielectric constant, $\epsilon_{r}$ of the material. The value of $\epsilon_{r}$ used in the prediction will depended on the materials of each individual building but it is often not very convenient to classify each building separately. Therefore a generally accepted practice is to use a single value of $\epsilon_{r}$ throughout the prediction area ranging between 3-15 [4], [5].

The actual value used in the prediction of the path loss plays an important but not so obvious role in the results when compared with the measurements. Figure 10 shows a plot of the predicted signals for $\epsilon_{r}=3,7$ and 12. A

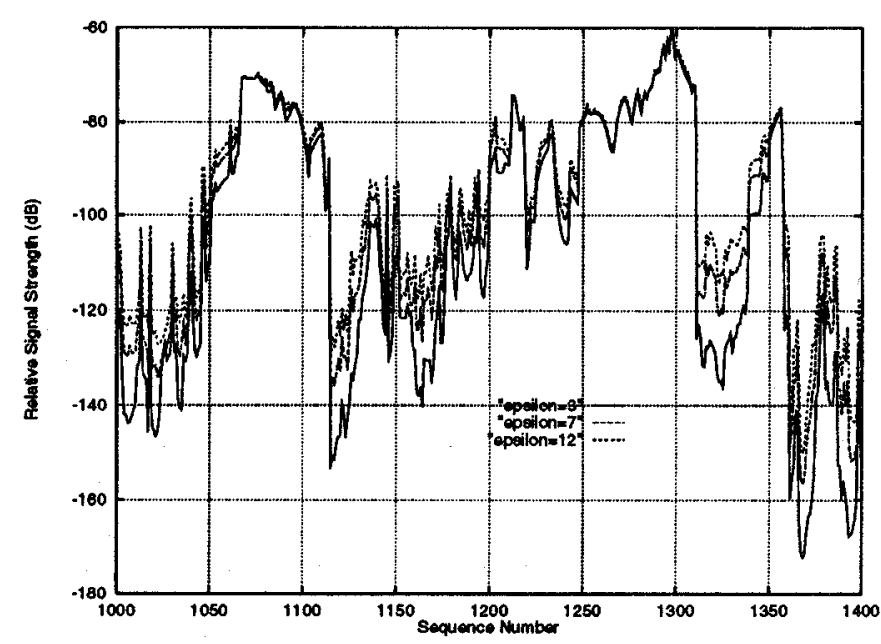

Fig. 10. Comparison of Site Specific Predictions for $3 \epsilon_{r}$ Values at Site 1a in Rosslyn

comparison of the curves shows that in the LOS and near LOS regions there is very little increase in the received signal even when the value of $\epsilon_{r}$ is quadrupled. On the other hand in the deeper shadows of the building where the predictions are generally more pessimistic than the measurement increasing the dielectric constant enhances the overall signal strength by as much as $20 \mathrm{~dB}$. Table I summarizes the statistical results when $\epsilon_{r}$ is increased from 3 to 15 . At $\epsilon_{r}=3$ the predictions are very pessimistic with 


\begin{tabular}{|c||c|c|c|c||}
\cline { 2 - 5 } \multicolumn{1}{c|}{} & $\epsilon_{r}=3$ & $\epsilon_{r}=7$ & $\epsilon_{r}=12$ & $\epsilon_{r}=15$ \\
\hline \hline$\eta$ & -12.0 & -4.3 & -0.8 & 0.4 \\
\hline$\sigma$ & 15.7 & 11.0 & 9.4 & 8.8 \\
\hline
\end{tabular}

TABLE I

The Prediction Accuracy With Different $\epsilon_{r}$ For tX1A IN ROSSLYN

a large average difference of $-12 \mathrm{~dB}$ and standard deviation of $15.7 \mathrm{~dB}$. As $\epsilon_{r}$ is gradually increased the average difference steadily decreased until it becomes slightly optimistic between $\epsilon_{r}=12$ and 15 , even as the standard deviation continues to decrease.

Figure 11 shows the predictions and measurements versus sequence number for the low antenna at Site $\mathrm{A}$, when

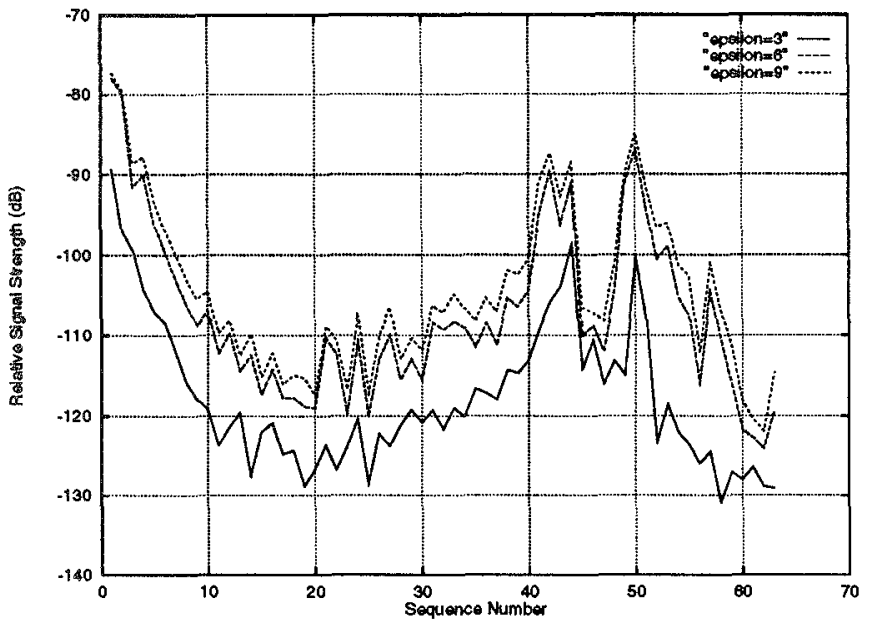

Fig. 11. Comparison of Site Specific Predictions for $3 \epsilon_{r}$ Values at Site A in Trenton

$\epsilon_{r}$ is 3,6 and 9 . When $\epsilon_{r}$ is 3 the average difference between the predictions and measurements is $-0.7 \mathrm{~dB}$, while the standard deviation is $4.9 \mathrm{~dB}$. For values of $\epsilon_{r}$ equal to 6 and 9 , the average difference is 9.7 and 12.55 , respectively. For this case the higher values of $\epsilon_{r}$ leads to predictions that are far too optimistic. This is in large part due to the contributions from paths around the buildings overwhelming the contributions from paths lying over the buildings.

Table II summarizes the prediction accuracy for the high and low antennas for both Sites A and B. The results show

\begin{tabular}{|c|c||c|c|c|c||}
\cline { 3 - 6 } \multicolumn{2}{c|}{} & \multicolumn{2}{c|}{ High Antenna } & \multicolumn{2}{c||}{ Low Antenna } \\
\cline { 2 - 6 } \multicolumn{2}{c|}{} & Site A & Site B & Site A & Site B \\
\hline \hline \multirow{3}{*}{$\eta(\mathrm{dB})$} & $\epsilon_{r}=3$ & -0.39 & -0.60 & -0.71 & -4.5 \\
\cline { 2 - 6 } & $\epsilon_{r}=6$ & 0.23 & 0.17 & 9.7 & 1.2 \\
\cline { 2 - 6 } & $\epsilon_{r}=9$ & 0.50 & 0.52 & 12.6 & 4.9 \\
\hline \multirow{3}{*}{$\sigma(\mathrm{dB})$} & $\epsilon_{r}=3$ & 4.5 & 4.8 & 4.9 & 7.7 \\
\cline { 2 - 6 } & $\epsilon_{r}=6$ & 4.8 & 4.8 & 5.7 & 7.0 \\
\cline { 2 - 6 } & $\epsilon_{r}=9$ & 4.9 & 4.9 & 5.6 & 7.0 \\
\hline
\end{tabular}

The Prediction Accuracy With Different $\epsilon_{r}$ for Trenton antenna increases while the standard deviation does not change appreciably. For the high antenna, when propagation is primarily over the buildings, the prediction error is seen to be insensitive to the values of $\epsilon_{r}$.

\section{Conclusions}

The results indicate that the theoretical site specific predictions that take into account building footprint and height are more accurate than using measurement based models. Moreover, accuracy of $1 \mathrm{~dB}$ average error and 4 to $9 \mathrm{~dB}$ standard deviation are achievable. The results of the comparisons of the average difference $\eta$ and standard deviation $\sigma$ are summarized in Table III for sites in Trenton, NJ and Rosslyn, VA.

\begin{tabular}{|l|c|c|c|c|c|c|}
\cline { 2 - 7 } \multicolumn{1}{c|}{} & \multicolumn{2}{c|}{$\begin{array}{c}\text { Trenton-Site A } \\
\text { High Antenna }\end{array}$} & \multicolumn{2}{c|}{$\begin{array}{c}\text { Trenton-Site A } \\
\text { Low Antenna }\end{array}$} & \multicolumn{2}{c|}{ Rosslyn } \\
\cline { 2 - 7 } & Fit & $\begin{array}{c}\text { Site } \\
\text { Specific }\end{array}$ & Fit & $\begin{array}{c}\text { Site } \\
\text { Specific }\end{array}$ & Fit & $\begin{array}{c}\text { Site } \\
\text { Specific }\end{array}$ \\
\hline$\eta(\mathrm{dB})$ & -4.8 & -0.39 & -5.7 & -0.7 & -3.4 & -0.8 \\
\hline$\sigma(\mathrm{dB})$ & 5.0 & 4.5 & 6.1 & 4.9 & 8.7 & 9.4 \\
\hline
\end{tabular}

Summary of the Prediction Accuracy Found in This Study

For the lower antenna height results given in Table 1, more ray paths must be accounted for, including reflections at buildings near to the base station preceding propagation over rooftops to the mobile's position, and diffraction around the corners of the buildings. These additional paths sometime give the dominant contribution.

\section{ACKNOWLEDGMENT}

The authors would like to thank Motorola for providing measurement data for Trenton NJ, and AT\&T for providing measurement data for for Rosslyn, VA.

\section{REFERENCES}

[1] H.H. Xia, H.L. Bertoni, L.R. Maciel, A. Lindsay-Stewart, and R. Rowe, "Microcellular Propagation Characteristics for Personal Communications in Urban and Suburban Environments", IEEE Transactions on Vehicular Technology, vol. 43, no. 3, pp. 743752, August 1994

[2] P.E. Mogensen, P. Eggers, C. Jensen, and J.B. Andersen, "Urban Area Radio Propagation Measurements at 955 and $1845 \mathrm{MHz}$ for Small and Micro Cells", in Proc. IEEE GLOBECOM'91, 1991, pp. 1297-1302.

[3] L.B. Felsen and N. Marcuvitz, Radiation and Scattering of Waves, Prentice-Hall, Inc, Englewood Cliffs, NJ, 1973.

[4] O. Landron, M.J. Feuerstein, and T.S. Rappaport, "A Comparison of Theoretical and Empirical Reflection Coefficients for Typical Exterior Wall Surfaces in Mobile Radio Environment", IEEE Transactions on Antennas and Propagation, vol. 44, no. 3, pp. 341-351, March 1996.

[5] H.R. Anderson, "A Ray-Tracing Propagation Model for Digital Broadcast Systems in Urban Areas", IEEE Transactions on Broadcasting, vol. 39, no. 3, pp. 309-317, September 1993. 